\title{
ON PRODUCTS OF HOCHSCHILD GROUPS
}

\author{
HISASHI YAMASAKI
}

(Received December 10, 1958)

Introduction. We shall consider three $K$-algebras $\Lambda, \Gamma, \Sigma$, where $K$ is a commutative ring, and that modules with operators are all unitary. ${ }_{\Lambda} A$ means a left $\Lambda$-module, and we transfer ${ }_{\Lambda} A$ to $A_{\Lambda^{*}}$ in many case, where $\Lambda^{*}$ is the "opposite" algebra of $\Lambda$. In the situation $\left(A_{\Lambda \otimes \Gamma},{ }_{\Lambda} B_{\Sigma}\right)^{1)}$, we convert $A \otimes{ }_{\Lambda} B$ into a right $\Gamma \otimes \Sigma$-module by setting

$$
(a \otimes b)(\gamma \otimes \sigma)=a \gamma \otimes b \sigma \text {. }
$$

Similarly in the situation $\left({ }_{\Lambda} B_{\Sigma}, C_{\Gamma \otimes \Sigma}\right)$ we convert $\operatorname{Hom}_{\Sigma}(B, C)$ into a right $\Lambda \otimes \Gamma$-module by setting

$$
(f(\lambda \otimes \gamma)) b=(f(\lambda b)) \gamma
$$

There are associative formulae:

(1) $r:\left(A \otimes_{\Lambda} B\right) \otimes_{\Gamma^{\prime} \otimes \Sigma} C \approx A \otimes_{\Lambda \otimes \Gamma}\left(B \otimes_{\Sigma} C\right), \quad \quad\left(A_{\Lambda_{\lrcorner} \Gamma,}, \quad{ }_{\Lambda} B_{\Sigma},{ }_{\Gamma \otimes \Sigma} C\right)$ such that $r[(a \otimes b) \otimes c]=a \otimes(b \otimes c)$,

(2) $s: \operatorname{Hom}_{\Lambda \otimes \mathrm{r}}\left(A, \operatorname{Hom}_{\Sigma}(B, C)\right) \approx \operatorname{Hom}_{\Gamma \otimes \Sigma}\left(A \otimes_{\Lambda} B, C\right), \quad\left(A_{\Lambda \otimes \Gamma},{ }_{\Lambda} B_{\Sigma}, C_{\Gamma \otimes \Sigma}\right)$ such that $(s f)(a \otimes b)=(f a) b$ for $f: A \rightarrow \operatorname{Hom}_{\Sigma}(B, C)$,

(3) $t: \operatorname{Hom}_{\mathrm{\Lambda} \otimes \mathrm{\Gamma}}\left(A, \operatorname{Hom}_{\Sigma}(B, C)\right) \approx \operatorname{Hom}_{\Sigma \otimes \mathrm{I}}\left(B \otimes_{\Lambda} A, C\right), \quad\left({ }_{\Lambda \otimes \Gamma} A,{ }_{\Sigma} B_{\Lambda}, \Sigma_{\Sigma} \otimes \mathrm{\Gamma} C\right)$ such that $(t g)(b \otimes a)=g(a) b$ for $g: A \rightarrow \operatorname{Hom}_{\Sigma}(B, C)$. (p. 165 of [1].)

Since these isomorphisms establish natural equivalences of functors, we get the transition of "projective" or "injective":

PROPOSITION 1 (pp. 165-6 of [1]). In the situation $\left(A_{\Lambda \otimes \Gamma},{ }_{\Lambda} B_{\Sigma}\right)$ assume that $A$ is $\Lambda \otimes \Gamma$-projective and $B$ is $\Sigma$-projective. Then $A \bigotimes_{\Lambda} B$ is projective as a right $\Gamma \otimes \Sigma$-module.

PROPOSITION 2 (loc. cit.). In the situation $\left({ }_{\Lambda} B_{\Sigma}, C_{\Gamma \otimes \Sigma}\right)$ assume that $B$ is $\Lambda$-projective and $C$ is $\Gamma \otimes \Sigma$-injective. Then $\operatorname{Hom}_{\Sigma}(B, C)$ is injective as a right $\Lambda \otimes \Gamma$-module.

Prop. 1 and Prop. 2 yield fundamental facts for the product theory, for instance,

PROPOSITION 3. (p. 166 of [1]). In the situation $\left(A_{\Lambda \otimes \Gamma},{ }_{\Lambda} B_{\Sigma}\right)$ let $X$ be a $\Lambda \otimes \Gamma$-projective resolution of $A$, and $Y$ a $\Lambda^{*} \otimes \Sigma$-projective resolution of $B$

1) $\otimes$ means tensor product over $K$; occasionally the mention of the rings of reference $K$ may be omitted when no confusion can occur. 
as a right $\Lambda^{*} \otimes \Sigma$-module. If $\operatorname{Tor}_{n}^{\prime}(A, B)=0$ for $n>0$ and $\Lambda, \Gamma, \Sigma$ are $K$-projective then $X \otimes_{1} Y$ is a $\Gamma \otimes \Sigma$-projective resolution of $A \bigotimes_{\mathrm{\Lambda}} B$.

For definitions of products, we specialised these propositions to the case $\Lambda=K, \cdots \cdots$ and applied two interchange homomorphisms : (p.64 and Chap. $\mathrm{XI}$ of $[1])$

$$
\begin{aligned}
& \boldsymbol{\alpha}: H^{p}(X) \otimes H^{q}(Y) \rightarrow H^{p_{+q}}(X \otimes Y), \\
& \boldsymbol{\alpha}^{\prime}: H^{p_{+} q}(\operatorname{Hom}(X, Y)) \rightarrow \operatorname{Hom}\left(H_{p}(X), H^{q}(Y)\right) .
\end{aligned}
$$

In this paper we shall define modified products $U, \cap$ for associative algebra (p. 228. Exercise 1. of [1]) and show that the reduction theorem of Hochschild groups for products $U$ and $\cap$ holds analogously to the case in groups. We obtain such and such by forcing only without the above specialisation $\Lambda=$ $K, \cdots \cdots$ in the section 1 . Further we shall translate this results into the relative Hochschild groups a little modified in the section 2 .

1. For the definitions of two products $\vee$ and $\wedge$ we make the following two assumptions :

(i) $\Lambda, \Gamma, \Sigma$ are $K$-projective.

(ii) $\operatorname{Tor}_{n}^{\Sigma}\left(A, A^{\prime}\right)=0$ for $n>0,{ }_{\Lambda} A_{\Sigma},{ }_{\Sigma} A_{\Gamma}^{\prime}$.

In the situation $\left({ }_{A} A_{\Sigma},{ }_{\Lambda} C_{\Sigma},{ }_{\Sigma} A_{\Gamma}^{\prime},{ }_{\Sigma} C_{\Gamma}^{\prime}\right)$, we define the $K$-homomorphism $\varphi_{3}: \operatorname{Hom}_{\Lambda \otimes \Sigma *}(A, C) \otimes \operatorname{Hom}_{\Sigma \otimes \Gamma *}\left(A^{\prime}, C^{\prime}\right) \rightarrow \operatorname{Hom}_{\Lambda \otimes \Gamma *}\left(A \otimes_{\Sigma} A^{\prime}, C \otimes_{\Sigma} C^{\prime}\right)$, by setting

$$
\left(\varphi_{3}\left(f \otimes f^{\prime}\right)\right)\left(a \otimes_{\Sigma} a^{\prime}\right)=f a \otimes_{\Sigma} f^{\prime} a^{\prime} .
$$

Replacing $A$ and $A^{\prime}$ by $X$ : a $\Lambda \otimes \Sigma^{*}$-projective resolution of $A$ and $X^{\prime}$ : a $\Sigma \otimes \Gamma^{*}$-projective resolution of $A^{\prime}$, we obtain

$$
\Phi_{3}: \operatorname{Hom}_{\Lambda \otimes \Sigma *}(X, C) \otimes \operatorname{Hom}_{\Sigma \otimes \Gamma *}\left(X^{\prime}, C^{\prime}\right) \rightarrow \operatorname{Hom}_{\Lambda \otimes \Gamma *}\left(X \otimes_{\Sigma} X^{\prime}, C \otimes_{\Sigma} C^{\prime}\right) \text {. }
$$

Passing to homology and applying $\alpha$, we obtain the homomorphism

$$
\operatorname{Ext}_{\Lambda \otimes \Sigma *}(A, C) \otimes \operatorname{Ext}_{\Sigma \otimes \Gamma_{*}}\left(A^{\prime}, C^{\prime}\right) \rightarrow H\left(\operatorname{Hom}_{\Lambda \otimes \mathrm{I} *}\left(X \otimes_{\Sigma} X^{\prime}, C \otimes_{\Sigma} C^{\prime}\right) .\right.
$$

On account of our assumptions (i), (ii), Prop. 3 shows that $X \otimes_{\mathrm{\Sigma}} X^{\prime}$ is a left $\Lambda \otimes \Gamma^{*}$-projective resolution of $A \otimes_{\Sigma} A^{\prime}$, therefore we get the modified $V$ product

$$
\vee: \operatorname{Ext}_{\Lambda \otimes \Sigma *}(A, C) \otimes \operatorname{Ext}_{\Sigma \otimes \Gamma *}\left(A^{\prime}, C^{\prime}\right) \rightarrow \operatorname{Ext}_{\Lambda \otimes \Gamma^{*}}\left(A \otimes_{\Sigma} A^{\prime}, C \otimes_{\Sigma} C^{\prime}\right) .
$$

Since $V$ is of degree zero, it yields maps

$$
\vee: \operatorname{Ext}_{\Lambda \otimes \Sigma *}^{p}(A, C) \otimes \operatorname{Ext}_{\Sigma \otimes \Gamma *}^{\eta}\left(A^{\prime}, C^{\prime}\right) \rightarrow \operatorname{Ext}_{\Lambda \otimes \Gamma *}^{p+q}\left(A \otimes_{\Sigma} A^{\prime}, C \otimes_{\Sigma} C^{\prime}\right) .
$$

For $p=q=0$ the $\vee$-product reduces to the homomorphism $\boldsymbol{\varphi}_{3}$.

Next in the situation $\left({ }_{\Lambda} A_{\Sigma},{ }_{\Lambda} C_{\Sigma},{ }_{\Sigma} A_{\Gamma}^{\prime},{ }_{\Gamma} C_{\Sigma}^{\prime}\right)$, we define $\operatorname{Hom}_{\Sigma}\left(C, C^{\prime}\right)$ as a right $\Lambda \otimes \Gamma^{*}$-module by

$$
\left[f\left(\lambda \otimes \gamma^{*}\right)\right] c=\gamma(f(\lambda c)), \quad c \in C, f \in \operatorname{Hom}_{\Sigma}\left(C, C^{\prime}\right),
$$

and $A \otimes_{\Sigma} A^{\prime}$ as a left $\Lambda \otimes \Gamma^{*}$-module by 


$$
\left(\lambda \otimes \gamma^{*}\right)\left(a \otimes a^{\prime}\right)=\lambda a \otimes a^{\prime} \gamma
$$

and the homomorphism

$$
\boldsymbol{\varphi}_{4}: \operatorname{Hom}_{\Sigma}\left(C, C^{\prime}\right) \otimes_{\mathbf{\Lambda} \otimes \Gamma^{*}}\left(A \otimes_{\Sigma} A^{\prime}\right) \rightarrow \operatorname{Hom}\left(\operatorname{Hom}_{\mathbf{\Lambda} \otimes \Sigma^{*}}(A, C), C^{\prime} \otimes_{\mathrm{\Sigma} \otimes \Gamma *} A^{\prime}\right),
$$

by setting

$\left(\varphi_{4}\left(f \otimes a \otimes a^{\prime}\right)\right) g=f(g a) \otimes a^{\prime}$, for $f \in \operatorname{Hom}_{\Sigma}\left(C, C^{\prime}\right), g \in \operatorname{Hom}_{\Lambda \otimes \Sigma *}(A, C)$, regarding $A$ and $C$ as left $\Lambda \otimes \Sigma^{*}$-modules, $A^{\prime}$ as a left $\Sigma \otimes \Gamma^{*}$-module and $C^{\prime}$ as a right $\Sigma \otimes \Gamma^{*}$-module. Replacing $A$ and $A^{\prime}$ by $X$ and $X^{\prime}$ we obtain

$$
\Phi_{4}: \operatorname{Hom}_{\Sigma}\left(C, C^{\prime}\right) \otimes_{\Lambda \otimes \Gamma *}\left(X \otimes_{\Sigma} X^{\prime}\right) \rightarrow \operatorname{Hom}\left(\operatorname{Hom}_{\Lambda \otimes \Sigma *}(X, C), C^{\prime} \otimes_{\Sigma \otimes \Gamma *} X^{\prime}\right) \text {. }
$$

Passing to homology and applying $\alpha^{\prime}$ we obtain the homomorphism

$$
H\left(\operatorname{Hom}_{\Sigma}\left(C, C^{\prime}\right) \otimes_{\Lambda \otimes \Gamma *}\left(X \otimes_{\Sigma} X^{\prime}\right)\right) \rightarrow \operatorname{Hom}\left(\operatorname{Ext}_{\Delta \otimes \Sigma *}(A, C), \operatorname{Tor}^{\Sigma \otimes \Gamma *}\left(C^{\prime}, A^{\prime}\right)\right) \text {. }
$$

Quite analogously to the case $\vee$ we obtain the $\wedge$ product

$$
\wedge: \operatorname{Tor}^{\Lambda \otimes \Gamma^{*}}\left(\operatorname{Hom}_{\Sigma}\left(C, C^{\prime}\right), A \otimes_{\Sigma} A^{\prime}\right) \rightarrow \operatorname{Hom}\left(\operatorname{Ext}_{\Lambda \otimes \Sigma *}(A, C), \operatorname{Tor}^{\Sigma \otimes \Gamma^{*}}\left(C^{\prime}, A^{\prime}\right)\right) \text {. }
$$

Since $\wedge$ has degree zero, it yields maps

$$
\wedge: \operatorname{Tor}_{p+g}^{\Delta \otimes \Gamma *}\left(\operatorname{Hom}_{\Sigma}\left(C, C^{\prime}\right), A \bigotimes_{\Sigma} A^{\prime}\right) \rightarrow \operatorname{Hom}\left(\operatorname{Ext}_{\Lambda}^{p}(A, C), \operatorname{Tor}_{q}^{\Gamma}\left(C^{\prime}, A^{\prime}\right)\right) \text {. }
$$

If $p=q=0$, the $\wedge$ product induces the map $\boldsymbol{\varphi}_{4}$.

We shall give the reduction theorem of these modified products. If $\Lambda=$ $\Gamma=\Sigma$ in the above, $A, A^{\prime}, C, C^{\prime}$ reduce to two-sided $\Lambda$-modules and we have (7) $\vee: \operatorname{Ext}_{\Lambda \otimes \Lambda *}(A, C) \otimes \operatorname{Ext}_{\Lambda \otimes \Lambda *}\left(A^{\prime}, C^{\prime}\right) \rightarrow \operatorname{Ext}_{\Lambda \otimes \Lambda *}\left(A \otimes_{\Lambda} A^{\prime}, C \otimes_{\Lambda} C^{\prime}\right)$, (8) $\wedge: \operatorname{Tor}^{\Lambda \otimes \Lambda *}\left(\operatorname{Hom}_{\Lambda}\left(C, C^{\prime}\right), A \otimes_{\Lambda} A^{\prime}\right) \rightarrow \operatorname{Hom}\left(\operatorname{Ext}_{\Lambda \otimes \Lambda *}(A, C), \operatorname{Tor}^{\Lambda \otimes \Lambda *}\left(C^{\prime}, A^{\prime}\right)\right.$. Case $\vee$. When $A=\Lambda$, $\operatorname{Tor}^{\Lambda}\left(\Lambda, A^{\prime}\right)=0$, for $n>0$, for any $A^{\prime}$, so that the assumptions (i), (ii) are satisfied and we can define a product

(9) $\vee: \operatorname{Ext}_{\Lambda \otimes \Lambda *}(\Lambda, C) \otimes \operatorname{Ext}_{\Lambda \otimes \Lambda *}\left(A^{\prime}, C^{\prime}\right) \rightarrow \operatorname{Ext}_{\Lambda \varangle \Lambda^{*}}\left(A^{\prime}, C \otimes_{\Lambda} C^{\prime}\right)$.

Using a two-sided $\Lambda$-module $B$, we define $\operatorname{Hom}_{\Lambda}\left(C^{\prime}, B\right)$, which is the group of right $\Lambda$-homomorphisms, as a left $\Lambda \otimes \Lambda^{*}$-module or as $\Lambda$-two-sided module by

$$
\left[\left(\lambda_{1} \otimes \lambda_{2}^{*}\right) f\right]\left(c^{\prime}\right)=\left[\lambda_{1} f \lambda_{2}\right]\left(c^{\prime}\right)=\lambda_{1}\left[f\left(\lambda_{2} c^{\prime}\right)\right]
$$

then the $K$-homomorphism of the left $\Lambda \otimes \Lambda^{*}$-module $\operatorname{Hom}_{\Lambda}\left(C^{\prime}, B\right) \otimes_{\Lambda} C^{\prime}$ into $B$

$$
\boldsymbol{\varphi}: \operatorname{Hom}_{\Lambda}\left(C^{\prime}, B\right) \otimes_{\Lambda} C^{\prime} \rightarrow B
$$

defined by

$$
\varphi\left(f \otimes_{\Lambda} c^{\prime}\right)=f\left(c^{\prime}\right),
$$

is a left $\Lambda \otimes \Lambda^{*}$-homomorphism. In fact,

$$
\begin{aligned}
\varphi\left[\left(\lambda_{1} \otimes \lambda_{2}^{*}\right)\left(f \otimes_{1} c^{\prime}\right)\right] & =\varphi\left[\left(\lambda_{1} f\right) \otimes c^{\prime} \lambda_{2}\right]=\left(\lambda_{1} f\right)\left(c^{\prime} \lambda_{2}\right)=\lambda_{1}\left[f\left(c^{\prime} \lambda_{2}\right)\right] \\
& =\lambda_{1} f\left(c^{\prime}\right) \lambda_{2}=\left(\lambda_{1} \otimes \lambda_{2}^{*}\right) \varphi\left(f \otimes_{1} c^{\prime}\right) .
\end{aligned}
$$

Now we may set $C=\operatorname{Hom}_{\Lambda}\left(C^{\prime}, B\right)$ in (9), and joining (9) with the homo- 
morphism induced by $\varphi$ yields a modified product of Hochschild groups (12) $\cup: H^{p}\left(\Lambda, \operatorname{Hom}_{\Lambda}\left(C^{\prime}, B\right)\right) \otimes \operatorname{Ext}_{\Lambda \otimes \Lambda *}^{q}\left(A^{\prime}, C^{\prime}\right) \rightarrow \operatorname{Ext}_{\Lambda \otimes \Lambda *}^{p+q}\left(A^{\prime}, B\right)$.

Case $\wedge$. When $A^{\prime}=\Lambda$, $\operatorname{Tor}_{n}^{\wedge}(A, \Lambda)=0, n>0$, for any $A$, so that the assumptions (i), (ii) are satisfied and we can define a product

(13) $\wedge: \operatorname{Tor}^{\Lambda \otimes \Lambda *}\left(\operatorname{Hom}_{\Lambda}\left(C, C^{\prime}\right), A\right) \rightarrow \operatorname{Hom}\left(\operatorname{Ext}_{\Lambda \otimes \Lambda *}(A, C), \operatorname{Tor}^{\Lambda \otimes \Delta *}\left(C^{\prime}, \Lambda\right)\right)$.

Using a two-sided $\Lambda$-module $B \otimes_{\Lambda} C$, we define the homomorphism

$$
\psi: B \rightarrow \operatorname{Hom}_{\Lambda}\left(C, B \otimes_{\Lambda} C\right) \text {, }
$$

by

$$
(\psi b)(c)=b \otimes_{\Lambda} c,
$$

where $\operatorname{Hom}_{\Lambda}\left(C, B \bigotimes_{\Lambda} C\right)$ is the groups of right $\Lambda$-homomorphisms, then

$$
\begin{aligned}
& \psi\left(b\left(\lambda_{1} \otimes \lambda_{2}^{*}\right)\right)(c)=\psi\left(\lambda_{2} b \lambda_{1}\right)(c)=\lambda_{2} b \lambda_{1} \otimes \bigotimes_{1} c=\lambda_{2} b \otimes \lambda_{1} c, \\
& {\left[\psi(b)\left(\lambda_{1} \otimes \lambda_{2}^{*}\right)\right](c)=\lambda_{2}\left[b \otimes\left(\lambda_{1} c\right)\right]=\lambda_{2} b \otimes \lambda_{1} c,}
\end{aligned}
$$

therefore $\psi$ is a right $\Lambda \otimes \Lambda^{*}$-homomorphism. Now we may set $C^{\prime}=B \otimes{ }_{\Lambda} C$ in (8), then $\psi$ and (13) induce a modified product

$$
\cap: \operatorname{Tor}_{p+q}^{\Lambda \otimes \Lambda^{*}}(B, A) \rightarrow \operatorname{Hom}\left(\operatorname{Ext}_{\Lambda \otimes \Lambda} *(A, C), H_{q}\left(\Lambda, B \otimes_{\Lambda} C\right)\right) \text {. }
$$

Rewriting (12) and (15), for two-sided $\Lambda$-modules $A, C, F$,

$$
\begin{aligned}
& U: H^{p}\left(\Lambda, \operatorname{Hom}_{\Lambda}(A, C)\right) \otimes \operatorname{Ext}_{\Lambda \otimes \Lambda^{*}}^{q}(F, A) \rightarrow \operatorname{Ext}_{\Lambda \otimes \Lambda^{*}}^{p+q}(F, C), \\
& \cap: \operatorname{Tor}_{p+q}^{\Lambda \otimes \Lambda^{*}}(C, F) \rightarrow \operatorname{Hom}\left(\operatorname{Ext}_{\Lambda \otimes \Lambda^{*}}^{p}(F, A), H_{q}\left(\Lambda, C \otimes_{\Lambda} A\right)\right) .
\end{aligned}
$$

When $F=A$ in (16), (17), using $j$ : the identity element of $A$ of $\operatorname{Hom}_{\Lambda \otimes \Lambda *}(A, A)$ $=\operatorname{Ext}_{\boldsymbol{\Lambda} \otimes \Lambda^{*}}^{0}(A, A)$, we define maps

$$
\begin{aligned}
& \cup j: H^{p}\left(\Lambda, \operatorname{Hom}_{\Lambda}(A, C)\right) \rightarrow \operatorname{Ext}_{\Lambda \otimes \Lambda^{*}}^{p}(A, C), \\
& \left.\cap j: \operatorname{Tor}_{p}^{\Lambda \otimes \Lambda^{*}}(C, A) \rightarrow H_{p}\left(\Lambda, C \bigotimes_{\Lambda} A\right)\right) .
\end{aligned}
$$

PROPOSITION 4. For $p=0$, the maps (17), (18) reduce to the isomorphism

$$
\begin{aligned}
& \sigma: \operatorname{Hom}_{\Lambda \otimes \Lambda^{*}}\left(\Lambda, \operatorname{Hom}_{\Lambda}(A, C)\right) \approx \operatorname{Hom}_{\Lambda \otimes \Lambda^{*}}(A, C), \\
& \tau: C \otimes_{\Lambda \otimes \Lambda^{*}} A \approx\left(C \otimes_{\Lambda} A\right) \bigotimes_{\Lambda \otimes \Lambda^{*}} \Lambda,
\end{aligned}
$$

given by

$\sigma(f)(a)=f(1)(a), \tau(c \otimes a)=(c \otimes a) \otimes 1$, where 1 is the unit element of $\Lambda$, which is $K$ projective.

This is a consequence of the definition of modified products and isomorphisms $r$ and $t$, and the fact that the left Hochschild group is right one.

PROPOSITION 5. If $A$ is right $\Lambda$-projective then $\cup j$ and $\cap j$ are isomorphisms, assume that $\Lambda$ is $K$-projective.

PROOF. Assuming that $U j$ and $\cap j$ are isomorphisms in degree $p(p \geqq 0)$ we shall prove for $p+1$. Consider a left $\Lambda \otimes \Lambda^{*}$-, that is two-sided $\Lambda$-. 
exact sequence $0 \rightarrow \mathrm{C} \rightarrow \mathrm{Q} \rightarrow \mathrm{N} \rightarrow 0$ with $Q$ left $\Lambda \otimes \Lambda^{*}$-injective. Since $A$ is right $\Lambda$-projective, the sequence of modules of right $\Lambda$-homomorphisms

$$
0 \rightarrow \operatorname{Hom}_{\Lambda}(A, C) \rightarrow \operatorname{Hom}_{\Lambda}(A, Q) \rightarrow \operatorname{Hom}_{\Lambda}(A, N) \rightarrow 0
$$

is left $\Lambda \otimes \Lambda^{*}$-exact, and $\operatorname{Hom}_{\Lambda}(A, Q)$ is right $\Lambda^{*} \otimes \Lambda$-, that is left $\Lambda \otimes \Lambda^{*}$. injective from the definition (10). Using the rules for commutation of $U$ with connecting homomorphisms, we obtain a commutative diagram

$$
\begin{aligned}
& H^{p}\left(\Lambda, \underset{\downarrow \cup_{j}}{\operatorname{Hom}}(A, Q)\right) \rightarrow H^{p}\left(\Lambda, \underset{\downarrow \cup_{j}}{\operatorname{Hom}}(A, N)\right) \rightarrow H^{p+1}(\Lambda, \underset{\uparrow \cap}{\operatorname{Hom}}(A, C)) \rightarrow 0 \\
& \operatorname{Ext}_{\Lambda \otimes \Lambda^{*}}^{p}(A, Q) \rightarrow \operatorname{Ext}_{\Lambda \otimes_{\Lambda^{*}}}^{p}(A, N) \rightarrow \operatorname{Ext}_{\Lambda \otimes \Lambda^{*}}^{p+1}(A, C) \rightarrow 0
\end{aligned}
$$

with exact rows. Since the first two vertical maps are isomorphisms the same follows for the third vertical map. The proof for $\cap j$ is similar using an exact sequence with $P \Lambda^{*} \otimes \Lambda$-projective.

Consider an exact sequence

$$
0 \rightarrow A \rightarrow F_{q-1} \rightarrow \cdots \cdots \rightarrow F_{0} \rightarrow F \rightarrow 0
$$

of left $\Lambda \otimes \Lambda^{*}$-modules $(q>0)$. The iterated connecting homomorphism

$$
\delta_{s}: \operatorname{Hom}_{\Lambda \otimes_{\Lambda^{*}}}(A, A) \rightarrow \operatorname{Ext}_{\Lambda \otimes_{\Lambda^{*}}}(F, A)
$$

is then defined. The image $\delta_{s} j \in \operatorname{Ext}_{\Lambda 8_{\Lambda^{*}}}(F, A)$ of the element $j$ is called the characteristic element of $(S)$.

The products (16) and (17) yield maps

$$
\begin{array}{ll}
\gamma: H^{p}\left(\Lambda, \operatorname{Hom}_{\Lambda}(A, C)\right) \rightarrow \operatorname{Ext}_{\Lambda \otimes^{*}}^{p+g^{*}}(F, C), & { }_{\Lambda} C_{\Lambda}, \\
\theta: \operatorname{Tor}^{\Lambda \varangle \Lambda^{*}}\left(C^{\prime} . F\right) \rightarrow H_{p}\left(\Lambda, C^{\prime} \otimes^{\Lambda} A\right), & { }_{\Lambda}^{\prime} C_{\Lambda}^{\prime},
\end{array}
$$

given by

$$
\begin{aligned}
& \gamma h=(-1)^{p q} h \cup \delta_{s} j, \\
& \theta h^{\prime}=h^{\prime} \cap \delta_{s} j, \quad h^{\prime} \in \operatorname{Tor}_{p+g}^{\Lambda \otimes \Delta^{*}}\left(C^{\prime}, F\right) \text {. }
\end{aligned}
$$

THEOREM 1 (Reduction Theorem). If $F_{0}, \cdots \cdots, F_{q-1}$ are left $\Lambda \otimes \Lambda^{*}$ projective and ${ }_{\Lambda{ }_{\Lambda}{ }^{*}} A={ }_{\Lambda} A_{\Lambda}$ is right $\Lambda$-projective, then maps $\gamma$ and $\theta$ are isomorphisms for $p>0$. For $p=0$ we have the exact sequence

$\operatorname{Hom}_{\Lambda \otimes^{*}}\left(F_{q-1}, C\right) \rightarrow \operatorname{Hom}_{\Lambda \otimes_{\Lambda^{*}}}(A, C) \rightarrow \operatorname{Ext}_{\Lambda \otimes^{*}}^{q}(F, C) \rightarrow 0$, $0 \rightarrow \operatorname{Tor}_{q}^{\Lambda \otimes_{\Lambda}^{*}}\left(C^{\prime}, F\right) \rightarrow C^{\prime} \bigotimes_{\Lambda \otimes_{\Lambda^{*}}} A \rightarrow C^{\prime} \bigotimes_{\Lambda \otimes_{\Lambda}^{*}} F_{q-1}$, where $\Lambda$ is $K$ projective.

Proof is quite similar to that of Theorem 9. 4. in p, 227 of [1].

COROLLARY 1.1. Let $\Lambda$ be $K$-projective, and

$$
0 \rightarrow F_{q}=A \rightarrow F_{q-1} \rightarrow \cdots \cdots \rightarrow F_{0} \rightarrow \Lambda \rightarrow 0, \quad q>0,
$$

be an exact sequence of left $\Lambda \otimes \Lambda^{*}$-modules with $F_{q-1}, \cdots \cdots, F_{0} \Lambda \otimes \Lambda^{*}$-projective. Then for $p>0$ we have

$$
H^{p}\left(\Lambda, \operatorname{Hom}_{\Lambda}(A, C)\right) \approx H^{p+q}(\Lambda, C),
$$




$$
H_{p+q}\left(\Lambda, C^{\prime}\right) \approx H_{p}\left(\Lambda, C^{\prime} \otimes_{\Lambda} A\right) .
$$

For $p=0$ we have the exact sequences

$$
\begin{aligned}
& \operatorname{Hom}_{\Lambda \otimes_{\Lambda^{*}}}\left(F_{q-1}, C\right) \rightarrow \operatorname{Hom}_{\Lambda \otimes_{\Lambda^{*}}}(A, C) \rightarrow H_{q}(\Lambda, C) \rightarrow 0, \\
& 0 \rightarrow H_{q}\left(\Lambda, C^{\prime}\right) \rightarrow C^{\prime} \otimes_{\Lambda \otimes_{\Lambda^{*}}} A \rightarrow C^{\prime} \otimes_{\Lambda \otimes_{\Lambda^{*}}} F_{q-1} .
\end{aligned}
$$

PROOF. Suppose that $M_{i}$ denote the image of $F_{i} \rightarrow F_{i-1}$, hence $M_{0}=\Lambda$. This yields an exact left $\Lambda \otimes \Lambda^{*}$-sequence

$$
0 \rightarrow M_{i-1} \rightarrow F_{i} \rightarrow M_{i} \rightarrow 0,
$$

in particular,

$$
0 \rightarrow M_{1} \rightarrow F_{0} \rightarrow \Lambda \rightarrow 0
$$

is left $\Lambda \otimes \Lambda^{*}$-exact and splits as a right $\Lambda$-sequence, so $M_{1}$ is a direct summand of $F_{0}$ as a right $\Lambda$-module. Since $F_{0}$ is left $\Lambda \otimes \Lambda^{*}$-projective and $\Lambda$ is $K$-projective, Prop. 1 yields that $F_{0}$ reduces to left $\Lambda^{*}$-projective, that is right $\Lambda$-projective, hence $M_{1}$ is right $\Lambda$-projective. Iterating this process, $A$ reduces to be right $\Lambda$-projective, and Cor. follows directly from Theorem 1.

Let $\Lambda$ be a $K$-algebra and $C$ a two-sided $\Lambda$-module. An extension over $\Lambda$ with kernel $C$ is an exact sequence

$$
\stackrel{\stackrel{g}{\rightarrow}}{\rightarrow} \stackrel{f}{\rightarrow} \Lambda
$$

where $\boldsymbol{\Gamma}$ is a $K$-algebra, $f$ is a $K$-algebra epimorphisms, $g$ is a monomorphism of $K$-modules and

$$
g(\lambda c)=\gamma(g c), g(c \lambda)=(g c) \gamma, \quad c \in C, \gamma \in \Gamma, \lambda=f \gamma \in \Lambda .
$$

Then we may assume that $\Gamma$ as a $K$-module coincides with the direct sum $\Gamma=C+\Lambda$, and that

$$
g_{c}=(c, 0), \quad f(c, \lambda)=\lambda .
$$

The multiplication in $\Gamma$ has the form

$$
\left(c_{1}, \lambda_{1}\right)\left(c_{2}, \lambda_{2}\right)=\left(c_{1} \lambda_{2}+\lambda_{1} c_{1}+a\left(\lambda_{1}, \lambda_{2}\right), \lambda_{1} \lambda_{2}\right) .
$$

The function $a$ is a $K$-homomorphism $a: \Lambda \otimes \Lambda \rightarrow C$ and may be regarded as a 2-cochain in the standard complex $S(\Lambda)$ with coefficients in $C$.

THEOREM 2 (G. Hochschild [3]). The set $F(\Lambda, C)$ of all equivalence classes of extensions over $\Lambda$ with kernel $C$ is in a 1-1-correspondence with the group $H^{2}(\Lambda, C)$. This correspondence $\omega: H^{2}(\Lambda, C) \rightarrow F(\Lambda, C)$ is obtained by assigning to each cocycle $a \in Z^{2}(S(\Lambda), C)$, the extension given by the multiplication (27). The inessential extensions form a single class of $F(\Lambda, C)$ and correspond to the zero element of $H^{2}(\Lambda, C)$.

Now let $\left\{u_{\lambda}\right\}$ be a set in a 1-1-correspondence with the set obtained by excluding $K$ from $\Lambda$, and $J$ be a left $\Lambda$-module, of which basis is $\left\{u_{\lambda}\right\}$ 
and $u_{k}=0$ for all $k \in K$. Suppose that $X$ is a direct sum of $J$ and $C$ as a left $\Lambda$-module, then we shall consider $X$ as a left $\Lambda \otimes \Lambda^{*}$-, that is two-sided $\Lambda$-module as follows. We give $C$ its former $\Lambda$-operations and define a right $\Lambda$-operation of $u_{\lambda}$ by setting

$$
\left(u_{\lambda_{1}}\right) \lambda_{2}=u_{\lambda_{1} \lambda_{2}}-\lambda_{1} u_{\lambda_{2}}+a\left(\lambda_{1}, \lambda_{2}\right), \quad \lambda_{1}, \lambda_{2} \in \Lambda,
$$

where $a\left(\lambda_{1}, \lambda_{2}\right)$ is a $2 \cdot$ cocycle corresponding to the extension $(F)$.

If Coker $K \rightarrow \Lambda$ is $K$-projective, we may assume that $a\left(\lambda_{1}, \lambda_{2}\right)$ is normalised, that is,

$$
a(\lambda, k)=a(k, \lambda)=0, k \in K . \quad \text { (p.176 of [1].) }
$$

Then, by setting $\lambda_{1}=k$ or $\lambda_{2}=k$ in (28), we have

$$
k u_{\lambda}=u_{k \lambda}=u_{\lambda k}=u_{\lambda} k \text {, }
$$

that is, $X$ may be considered as a $K$-module. Furthermore,

$$
\begin{aligned}
\lambda_{1}\left(u_{\lambda} \lambda_{2}\right) & =\lambda_{1}\left(u_{\lambda_{\lambda 2}}-\lambda u_{\lambda_{2}}+a\left(\lambda, \lambda_{2}\right)\right)=\lambda_{1} u_{\lambda \lambda_{2}}-\left(\lambda_{1} \lambda\right) u_{\lambda_{2}}+\lambda_{1} a\left(\lambda, \lambda_{2}\right) \\
& =u_{\lambda_{1} \lambda_{2}}-\left(u_{\lambda_{1}}\right) \lambda \lambda_{2}+a\left(\lambda_{1}, \lambda \lambda_{2}\right)-u_{\lambda_{1} \lambda_{2}}+u_{\lambda_{1} \lambda} \lambda_{2}-a\left(\lambda_{1} \lambda, \lambda_{2}\right)+\lambda_{1} a\left(\lambda, \lambda_{2}\right) \\
& =u_{\lambda_{1} \lambda} \lambda_{2}-\left(u_{\lambda_{1}}\right) \lambda \lambda_{2}+a\left(\lambda_{1}, \lambda\right) \lambda_{2}=\left[u_{\lambda_{1} \lambda}-u_{\lambda_{1}} \lambda+a\left(\lambda_{1}, \lambda\right)\right] \lambda_{2} \\
& =\left(\lambda_{1} u_{\lambda}\right) \lambda_{2},
\end{aligned}
$$

hence $X$ becomes a two-sided $\Lambda$-module.

Next we define the $K$-homomorphisms

$$
\pi: X \rightarrow \Lambda \otimes \Lambda^{*}
$$

by setting

$$
\pi(c)=0, \pi\left(u_{\lambda}\right)=\lambda \otimes 1-1 \otimes \lambda^{*}, \quad \pi\left(\lambda_{1} u_{\lambda}\right)=\lambda_{1} \pi\left(u_{\lambda}\right),
$$

then

$$
\begin{aligned}
\pi\left(u_{\lambda} \lambda_{2}\right) & =\pi\left(u_{\lambda \lambda_{2}}-\lambda u_{\lambda_{2}}+a\left(\lambda, \lambda_{2}\right)\right)=\lambda \lambda_{2} \otimes 1-1 \otimes \lambda_{2}^{*} \lambda^{*}-\lambda\left(\lambda_{2} \otimes 1-1 \otimes \lambda_{2}^{*}\right) \\
& =\lambda \otimes \lambda_{2}^{*}-1 \otimes \lambda_{2}^{*} \lambda^{*}=\left(1 \otimes \lambda_{2}^{*}\right)\left(\lambda \otimes 1-1 \otimes \lambda^{*}\right)=\left(1 \otimes \lambda_{2}^{*}\right) \pi\left(u_{\lambda}\right),
\end{aligned}
$$

therefore, regarding $X$ as a left $\Lambda \otimes \Lambda^{*}$-module, $\pi$ is a left $\Lambda \otimes \Lambda^{*}$-homomorphism, and $C$ is the kernel of $\pi$. Let $I$ be the kernel of the argumentation

$$
\rho: \Lambda \otimes \Lambda^{*} \rightarrow \Lambda
$$

given $\rho\left(\mu \otimes \gamma^{*}\right)=\mu \gamma$, regarding $\Lambda$ as a left $\Lambda \otimes \Lambda^{*}$-module with operators

$$
\left(\mu \otimes \gamma^{*}\right) \lambda=\mu \lambda \gamma, \quad \lambda, \mu, \gamma \in \Lambda \text {, }
$$

then $I$ is generated by the elements $\left(\lambda \otimes 1-1 \otimes \lambda^{*}\right)$, (p.168 of [1]), therefore $\pi$ is an epimorphism onto $I$ and we have a left $\Lambda \otimes \Lambda^{*}$-extension over $I$ with kernel $C$

$$
0 \rightarrow C \rightarrow X \rightarrow I \rightarrow 0 .
$$

Thus we obtain a mapping between extention classes 


$$
\eta: F(\Lambda, C) \rightarrow E(I, C) .
$$

Since $a\left(\lambda_{1}, \lambda_{2}\right)$ reduces to coboundary regarding as a 2 -cocycle with coefficients $X$, the corresponding extension over $\Lambda$ with kernel $X$ is inessential.

For $\boldsymbol{\Gamma}$ : the extension determined by the cocycle $a\left(\lambda_{1}, \lambda_{2}\right)$, we define a map

$$
l: \Gamma \rightarrow X
$$

by setting

$$
l(c, \lambda)=c+u_{\lambda}
$$

then,

$$
\begin{aligned}
l\left[\left(c_{1}, \lambda_{1}\right)\left(c_{2}, \lambda_{2}\right)\right] & =l\left(c_{1} \lambda_{2}+\lambda_{1} c_{2}+a\left(\lambda_{1}, \lambda_{2}\right), \lambda_{1} \lambda_{2}\right)=c_{1} \lambda_{2}+\lambda_{1} c_{2}+a\left(\lambda_{1}, \lambda_{2}\right)+u_{\lambda_{1} 2} \\
& =c_{1} \lambda_{2}+\lambda_{1} c_{2}+a\left(\lambda_{1}, \lambda_{2}\right)+\left(u_{\lambda_{1}}\right) \lambda_{2}+\lambda_{1} u_{\lambda_{2}}-a\left(\lambda_{1}, \lambda_{2}\right) \\
& =\left(c_{1}+u_{\lambda_{1}}\right) \lambda_{2}+\lambda_{1}\left(c_{2}+u_{\lambda_{2}}\right) \\
& =l\left(c_{1}, \lambda_{1}\right) f\left(c_{2}, \lambda_{1}\right)+f\left(c_{1}, \lambda_{1}\right) l\left(c_{2}, \lambda_{2}\right),
\end{aligned}
$$

therefore we have seen that

(34) The map $l$ is a 1-cocycle of $\Gamma$ with coefficients $X$, if we regard left $\Lambda \otimes \Lambda^{*}$-module $X$ as a left $\Gamma \otimes \Gamma^{*}$-module, using $f: \Gamma \rightarrow \Lambda$.

THEOREM 3. If $\Lambda$ is a K-projective algebra, such that Coker $K \rightarrow \Lambda$ is a K-projective module, then the following diagram is anticommutative.

$$
\begin{aligned}
& F(\Lambda, C) \stackrel{\omega^{-1}}{\longrightarrow} H^{2}(\Lambda, C)=\operatorname{Ext}^{2}{ }_{\Lambda \otimes \Lambda^{*}}(\Lambda, C) \\
& \eta \downarrow \downarrow \\
& E(I, C) \longrightarrow{ }_{\circledast x} \operatorname{Ext}_{\Lambda \otimes \Lambda^{*}}^{1}(I, C)
\end{aligned}
$$

where $\Theta$ is the correspondence between the $\Lambda \otimes \Lambda^{*}$-extension classes $\mathrm{Ext}_{\Lambda \otimes \Lambda^{*}}^{1}$ and $\mu$ is the connecting homomorphism corresponding to the exact sequence: $0 \rightarrow I \rightarrow \Lambda \otimes \Lambda^{*} \rightarrow \Lambda \rightarrow 0$.

Since $\mu$ is an isomorphism, and $\Theta$ and $\omega$ are 1-1, we obtain

COROLLARY 3.1. The correspondence (32) is 1-1.

PROOF. If we show $X$ is $\Lambda \otimes \Lambda^{*}$-projective, by joining (31) with the exact sequence : $0 \rightarrow I \rightarrow \Lambda \otimes \Lambda^{*} \rightarrow \Lambda \rightarrow 0$, the sequence

$$
0 \rightarrow C \rightarrow X \rightarrow \Lambda \otimes \Lambda^{*} \rightarrow \Lambda \rightarrow 0
$$

is left $\Lambda \otimes \Lambda^{*}$-exact, and we may assume that

$$
\cdots \cdots X_{1}=X \rightarrow X_{0}=\Lambda \otimes \Lambda^{*} \rightarrow \Lambda \rightarrow 0
$$

is a left $\Lambda \otimes \Lambda^{*}$-projective resolution of $\Lambda$. Then our assertion follows from the comparision of the characteristic element of $(E): \delta_{E} j$ and the image of $j$ by the iterated connecting homomorphism induced by the natural mapping $(R) \rightarrow$ the projective resolution of $C:(R): \cdots \cdots \rightarrow X_{2} \rightarrow C \rightarrow 0$, which maps 
the module $X_{n}$ of $(R)$ identically into the module $X_{n}$ of $(\underline{R})$ for $n \geqq 2$, and maps $X_{n}$ into zero for $n<2$. This map lowers the degree by 2 and commutes with the differentiation. (p. 92 of [1].)

We represent $K$-algebra $\Lambda$ as a residue class ring of a $K$-free algebra $F$ with kernel $R$, which is a ideal of $F$, and denote $C=R / R^{2}, \Gamma=F / R^{2}$, then we have obtained a $\Lambda \otimes \Lambda^{*}$-extension of $K$-algebra over with kernel $C$ :

$$
C \rightarrow \Gamma \stackrel{f}{\rightarrow} \Lambda, \quad C=R / R^{2}, \Gamma=F / R^{2},
$$

therefore it results that we have considered $R / R^{2}$ as a two-sided $\Lambda$-module by using the epimorphism $f$,

$$
\lambda c=\omega c, c \lambda=c \omega, \quad c \in C, \omega \in \Gamma, \lambda=f \omega \in \Gamma .
$$

Provided the theorem has been proved, the element $\xi$ of $H^{2}(\Lambda, C)$ corresponding to the extension $(\Sigma)$ is the negative of the characteristic element of $(E)$, therefore applied Cor. 1.1 for $(E)$, now $q=2$, we obtain

THEOREM 4. If $\Lambda$ is a K-projective algebra such that Coker $K \rightarrow \Lambda$ is $K$-projective, then for $\xi$ : the cohomology class determined by $(\Sigma)$, the following isomorphisms hold for $p>0$

$$
\begin{aligned}
& H^{p}\left(\Lambda, \operatorname{Hom}_{\Lambda}(C, A)\right) \approx H^{p+2}(\Lambda, A), \\
& H_{p+2}(\Lambda, A) \approx H_{p}\left(\Lambda, A \bigotimes_{\Lambda} C\right)
\end{aligned}
$$

given by the modified products $h \rightarrow h \cup \xi,\left(h \in H^{p}\left(\Lambda, \operatorname{Hom}_{\Lambda}(C, A)\right)\right.$ and $h^{\prime} \rightarrow$ $h^{\prime} \cap \xi\left(h^{\prime} \in H_{p+2}(\Lambda, A)\right)$. For $p=0$, we have exact sequences

$$
\begin{aligned}
& \operatorname{Hom}_{\Lambda 8_{\Lambda^{*}}}(\Gamma, \Lambda) \rightarrow \operatorname{Hom}_{\Lambda \otimes_{\Lambda^{*}}}(C, A) \rightarrow H^{2}(\Lambda, A) \rightarrow 0 \\
& 0 \rightarrow H_{2}(\Lambda, A) \rightarrow A \otimes_{\Lambda} C \rightarrow A \bigotimes_{\Lambda} \Gamma .
\end{aligned}
$$

REMARK. $\operatorname{Hom}_{\Lambda}(C, A)$ may be considered as the group $\operatorname{Hom}_{F}(R, A)$ of all right $F$-homomorphisms of $R$ into $A$ regarding $R$ and $A$ as $F$-modules induced by the map $n: F \rightarrow \Lambda$, tecause $k\left(r_{1} r_{2}\right)=k\left(r_{1}\right) n\left(r_{2}\right)=0$, so that $\left(r_{1} k\right)(r)$ $=n\left(r_{1}\right) k(r)=0$ and $\left(k r_{2}\right)(r)=k\left(r r_{2}\right)=0$, for $k \in \operatorname{Hom}_{F}(R, A)$.

To show that $X$ is $\Lambda \otimes \Lambda^{*}$-projective, whenever $\alpha: A \rightarrow B$ is a $\Lambda \otimes \Lambda^{*}$ epimorphism and $\beta: X \rightarrow B$ is a $\Lambda \otimes \Lambda^{*}$-homomorphism, it suffices to see that there is a $\Lambda \otimes \Lambda^{*}$-homomorphism $\gamma: X \rightarrow \mathrm{A}$, such that $\alpha \gamma=\beta$. First we shall define a map $h$ of $F$ into $A$ such that

$$
\alpha h=\beta l m, h\left(u_{1} u_{2}\right)=f m\left(u_{1}\right) h\left(u_{2}\right)+h\left(u_{1}\right) f m\left(u_{2}\right), \quad u_{1}, u_{2} \in F,
$$

where $m$ is the epimorphism of $F$ onto $\Gamma$.

In fact, taking $\left\{z_{\nu}\right\}$ a system of free generators, there is an element $a_{\nu}$ of $A$ such that $\alpha\left(a_{v}\right)=\beta \operatorname{lm}\left(z_{\nu}\right)$ because $\alpha$ is an epimorphism. Then we assign $h\left(z_{v}\right)=a_{\nu}$. In particular, since $l m k=0+u_{k}=0$ for $k \in K$, we may set 
$a_{\nu}=0$, hence $h(k)=0$, and so, if we set $u_{1}=k, u_{2}=1$, (38) is true for $k \in K$. Therefore applying the induction for the length of "words", we can define $h(z)$ on the whole $F$ so that the second of (38) may be satisfied.

Now assume that the first holds for $z_{\nu}$ and $z_{\mu}$, since $l$ is a 1 -cocycle as (34),

$$
\beta \operatorname{lm}\left(z_{\nu} z_{\nu}\right)=\beta\left[f m\left(z_{\nu}\right) \operatorname{lm}\left(z_{\mu}\right)+\operatorname{lm}\left(z_{v}\right) f m\left(z_{\mu}\right)\right],
$$

further since $\alpha, \beta$ are two-sided $\Lambda$-homomorphisms,

$$
\begin{aligned}
& =f m\left(z_{\nu}\right)\left[\beta \operatorname{lm}\left(z_{\mu}\right)\right]+\left[\beta \operatorname{lm}\left(z_{\nu}\right)\right] f m\left(z_{\mu}\right), \text { from the inductive assumption, } \\
& =f m\left(z_{\nu}\right)\left[\alpha h\left(z_{\mu}\right)\right]+\left[\alpha h\left(z_{\nu}\right)\right] f m\left(z_{\mu}\right) \\
& =\alpha\left[f m\left(z_{\nu}\right) h\left(z_{\mu}\right)+h\left(z_{\nu}\right) f m\left(z_{\mu}\right)\right] \\
& =\alpha h\left(z_{\nu} z_{\mu}\right) .
\end{aligned}
$$

Thus inductively we may define the desired map $h$ on the whole $F$, and since this $h$ is identically zero on $R^{2}$ because of the second of (38), we have also a map $h^{\prime}$ of $\Gamma$ into $A$ such that

$$
\alpha h^{\prime}=\beta l, h^{\prime}\left(\omega_{1} \omega_{2}\right)=f\left(\omega_{1}\right) h^{\prime}\left(\omega_{2}\right)+h^{\prime}\left(\omega_{1}\right) f\left(\omega_{2}\right), \quad \omega_{1}, \omega_{2} \in \Gamma .
$$

Finally we define a $K$-homomorphism $\gamma$ of $X$ into $A$ by setting

$$
\gamma(c)=h^{\prime}(c, 0), \quad \gamma\left(\lambda_{1} u_{\lambda}\right)=\lambda_{1} h^{\prime}(0, \lambda), \quad c \in C, \lambda, \lambda_{1} \in \Lambda,
$$

then

$$
\begin{array}{rlr}
\gamma\left(u_{\lambda} \lambda_{2}\right) & =\gamma\left(u_{\lambda \lambda_{2}}-\lambda u_{\lambda 2}+a\left(\lambda, \lambda_{2}\right)\right), & a\left(\lambda, \lambda_{2}\right) \in \xi \\
& =h^{\prime}\left(a\left(\lambda, \lambda_{2}\right), \lambda \lambda_{2}\right)-\lambda h^{\prime}\left(0, \lambda_{2}\right) & \\
& =h^{\prime}\left((0, \lambda),\left(0, \lambda_{2}\right)\right)-\lambda h^{\prime}\left(0, \lambda_{2}\right) \\
& =f(0, \lambda) h^{\prime}\left(0, \lambda_{2}\right)+h^{\prime}(0, \lambda) f\left(0, \lambda_{2}\right)-\lambda h^{\prime}\left(0, \lambda_{2}\right)=h^{\prime}(0, \lambda) \lambda_{2} \\
& =\left[\gamma\left(u_{\lambda}\right)\right] \lambda_{2}, &
\end{array}
$$

so that $\gamma$ is a two-sided $\Lambda$-homomorphism, and

$$
\alpha \gamma\left(c+u_{\lambda}\right)=\alpha h^{\prime}(c, \lambda)=\beta l(c, \lambda)=\beta\left(c+u_{\lambda}\right),
$$

consequently

$$
\alpha \gamma=\beta,
$$

this proves our assertions.

2. The Relative Case. We shall consider two $K$-rings $\Lambda$ and $\Gamma$ with a $K$-ring homomorphism $\boldsymbol{\varphi}: \Lambda \rightarrow \Gamma$. Then co (contra) variant $\boldsymbol{\phi}$-extensions, $\boldsymbol{\varphi}$ projective modules, $\varphi$-injective modules are defined and it holds that many identifications between these various extensions and many changes of “..-projective" or “..-injective”. (\$6. Chap. II of [1]). For example,

PROPOSITION 6. For any (right) $\Lambda$-module A, covariant $\phi$-extension of 
$A$ is $\boldsymbol{\varphi}$-projective and contravariant $\varphi$-extension of $A$ is $\varphi$-injective.

The first thing we shall define " $(\boldsymbol{\Gamma}, \boldsymbol{\Lambda})$-exact". An exact sequence of $\boldsymbol{\Gamma}$ homomorphisms between left $\Gamma$-modules $t_{i}: M_{i} \rightarrow M_{i-1}\left(\right.$ or $t^{i}: M^{i} \rightarrow M^{i+1}$ ) is called $(\Gamma, \Lambda)$-exact if for each $i$, the kernel of $t_{i}$ is a direct summand as a $\Lambda$-module induced by $\boldsymbol{\phi}$. (This definition reduces to that of [4], when $\Lambda$ is a $K$-subalgebra of $\boldsymbol{\Gamma}$ with the injection $i$ as $\varphi$.) A sequence of $\Gamma$-homomorphisms $t_{i}$ is $(\boldsymbol{\Gamma}, \boldsymbol{\Lambda})$-exact if and only if, (a) $t_{i} \circ t_{i+1}=0$, (b) there exists an $\Lambda$-homotopy, i.e., a sequence of $\Lambda$-homomorphisms $h_{i}: M_{i} \rightarrow M_{i+1}$, regarding $M_{i}$ as a $\Lambda$-module by $\varphi$, such that $t_{i+1}{ }^{\circ} h_{i}+h_{i-1}{ }^{\circ} t_{i}$ is the identity map of $M_{i}$ onto itself. Similar definitions are done for right modules.

If for every exact $(\Gamma, \Lambda)$-sequence $0 \rightarrow U \stackrel{p}{\rightarrow} V \stackrel{q}{\rightarrow} W \rightarrow 0$, and every $\Gamma$ homomorphism $g$ of $A$ into $W$, there is a $\Gamma$-homomorphism $g^{\prime}$ of $A$ into $V$ such that $q \circ g^{\prime}=g$, a $\Gamma$-module $A$ is said to be $(\Gamma, \Lambda)$-projective, dually if for every such sequence and every $\Gamma$-homomorphisms $h$ of $U$ into $C$, there is a $\Gamma$-homomorphism $h^{\prime}$ of $V$ into $C$, such that $h^{\prime} \circ p=h$, then a $\Gamma$-module $C$ is said to be $(\Gamma, \Lambda)$-injective.

PROPOSITION 7. " $\varphi$-projective" is equivalent to $(\Gamma, \Lambda)$-projective. " $\varphi$ injective" is equivalent to $(\Gamma, \Lambda)$-injective.

PROOF. Let $A_{\Gamma}$ be $(\Gamma, \Lambda)$-projective and $g$ be a $\Gamma$-homomorphism: $A_{(\varphi)}=A_{\Gamma} \otimes_{\Lambda} \Gamma \rightarrow A$ defined by $g(a \otimes \gamma)=a \gamma$. Since the sequence $0 \rightarrow \mathrm{Ker}$ $g \rightarrow A_{(\varphi)} \rightarrow A \rightarrow 0$ splits as a $\Lambda$-sequence, there exists $\Gamma$-homomorphism $g^{\prime}$ such that $g g^{\prime}$ is the identity of $A$, which proves that $A$ is $\boldsymbol{\varphi}$-projective. Conversely let $A$ be $\phi$-projective, and $h$ be a $\Gamma$-homomorphism of $A$ into $W$, seeing that $A_{\boldsymbol{\Gamma}} \bigotimes_{\boldsymbol{\Lambda}} \Gamma$ is always $(\boldsymbol{\Gamma}, \Lambda)$-projective (quite similarly to Lemma 2. of [4].), there exists a $\Gamma$-homomorphism $d$ such that $q d=h g$, hence $h=h g g^{\prime}=q\left(d g^{\prime}\right)$ for $A$, because $A$ is $\boldsymbol{\phi}$-projective, therefore $A$ is $(\Gamma, \Lambda)$ projective.

These facts may be quite formally obtained from the discussion of [4] only by the following translation: "Replacing $K$-ring $R$, its subring $S$, the injection $i: S \rightarrow R$, by $\Gamma, \Lambda, \varphi^{\prime \prime}$, therefore it may yield another relative (co) homology theory. In fact if we translate as above, the results in [4] hold together with proofs, hence from now on we shall refer to them freely with the appropriate modefications to our case. For instance we have easily just like to the usual balances,

PROPOSITION 8. $t_{i}: M_{i} \rightarrow M_{i-1}$ be any exact $(\Gamma, \Lambda)$ sequence, it follows that the induced sequence of homomorphisms $\operatorname{Hom}_{\Gamma}\left(M_{i-1}, A\right) \rightarrow \operatorname{Hom}_{\Gamma}\left(M_{i}, A\right)$, $h \rightarrow h \circ t_{i}$, is exact if and only if $A$ is $(\Gamma, \Lambda)$-injective. Similarly, the induced sequence of homomorphisms $\operatorname{Hom}_{\Gamma}\left(A, M_{i}\right) \rightarrow \operatorname{Hom}_{\Gamma}\left(A, M_{i-1}\right), h \rightarrow t_{i} \circ h$, 
is exact if and only if $A$ is $(\Gamma, \Lambda)$-projective.

To explain the reduction theorem in our relative case, we must begin with an analogy of Prop. 1. and Prop. 2.

Let $\Pi, P, \Lambda, \Gamma, T, \Sigma$ be $K$-algebras, with a $K$-ring homomorphism $\kappa: \Pi \rightarrow P$, and $\varphi: \Lambda \rightarrow \Gamma$, which yield a $K$-ring homomorphism : $\kappa \otimes \varphi: \Pi \otimes \Lambda \rightarrow P \otimes \Gamma$ defined by $(\kappa \otimes \varphi)(\pi \otimes \lambda)=\kappa(\pi) \otimes \varphi(\lambda)$, and with $\psi: T \rightarrow \Sigma$, which yield a $K$-ring homomorphism : $\kappa^{*} \otimes \psi: \Pi^{*} \otimes T \rightarrow P^{*} \otimes \Sigma$ defined by $\left(\kappa^{*} \otimes \psi\right)$ $\left(\pi^{*} \otimes \tau\right)=\kappa^{*}\left(\pi^{*}\right) \otimes \psi(\tau)$,

PROPOSITION 9. In the situation $\left(A_{P \ngtr \Gamma},{ }_{P} B_{\Sigma}\right)$ if $A$ is $(P \otimes \Gamma, \Pi \otimes \Lambda)$ projective then a right $\Gamma \otimes \Sigma$-module $A \otimes_{P} B$ is $(\Gamma \otimes \Sigma, \Lambda \otimes T)$-projective. In the situation $\left({ }_{\Sigma} B_{P}, C_{\Gamma \otimes P}\right)$ if $C$ is $(\Gamma \otimes P, \Lambda \otimes \Pi)$-injective then a right $\Sigma \otimes \Gamma$-module $\operatorname{Hom}_{P}(B, C)$ is $(\Sigma \otimes \Gamma, T \otimes \Lambda)$-injective.

PROOF. In the situation $\left(A_{P \otimes \mathrm{r}},{ }_{P} B_{\Sigma}\right)$, let $X_{\Gamma \otimes \Sigma}, Y_{\Gamma \otimes \Sigma}$ be right $\Gamma \otimes \Sigma$-modules, and let $\rho: X \rightarrow Y$ be a $\Lambda \otimes T$-homomorphism, regarded $X$ and $Y$ as $\Lambda \otimes T$ modules induced by $\varphi \otimes \psi$, then $\rho$ yields a map of ${ }_{P} B_{\Sigma}$,

$$
\rho^{\prime}: \operatorname{Hom}_{\Sigma}(B, X) \rightarrow \operatorname{Hom}_{\Sigma}(B, Y) \text {. }
$$

Considering these modules as right $\Pi \otimes \Lambda$-modules by

$$
(h \circ \pi) b=h(\kappa(\pi) b),(h \circ \lambda) b=h(b) \varphi(\lambda), h \in \operatorname{Hom}_{\Sigma}(B, \quad),
$$

it holds that for $h \in \operatorname{Hom}_{\Sigma}(B, X)$,

$$
\begin{aligned}
& {\left[\rho^{\prime}(h \circ \pi)\right] b=\rho[h(\kappa(\pi) b)]=\left(\rho^{\prime} h\right)(\kappa(\pi) b)=\left[\left(\rho^{\prime} h\right) \circ \pi\right] b,} \\
& {\left[\rho^{\prime}(h \circ \lambda)\right] b=\rho[h(b) \phi(\lambda)]=[\rho h(b)] \phi(\lambda)=\left[\left(\rho^{\prime} h\right) b\right] \phi(\lambda)=\left[\left(\rho^{\prime} h\right) \circ \lambda\right] b,}
\end{aligned}
$$

that is, $\rho^{\prime}$ is a $\Pi \otimes \Lambda$-homomorphism. Furthermore if $\rho$ is a $\Gamma \otimes \Sigma$-homomorphism from the beginning, it follows that in the same way $\rho^{\prime}$ is a $P \otimes \Gamma$ homomorphism.

Therefore, on account of the definition of our relative exactness (a),(b), for any exact $(\Gamma \otimes \Sigma, \Lambda \otimes T)$-sequence : $0 \rightarrow C^{\prime} \rightarrow C \rightarrow C^{\prime \prime} \rightarrow 0$ we have an exact $(P \otimes \Gamma, \Pi \otimes \Lambda)$-sequence

$$
0 \rightarrow \operatorname{Hom}_{\Sigma}\left(B, C^{\prime}\right) \rightarrow \operatorname{Hom}_{\Sigma}(B, C) \rightarrow \operatorname{Hom}_{\Sigma}\left(B, C^{\prime \prime}\right) \rightarrow 0
$$

Seeing that $A$ is $(P \otimes \Gamma, \Pi \otimes \Lambda)$-projective, this sequence yields from Prop. 8 an exact sequence

$$
\begin{aligned}
& 0 \rightarrow \operatorname{Hom}_{P \otimes \Gamma}\left(A, \operatorname{Hom}_{\Sigma}\left(B, C^{\prime}\right)\right) \rightarrow \operatorname{Hom}_{P \otimes \Gamma}\left(A, \operatorname{Hom}_{\Sigma}(B, C)\right) \\
& \rightarrow \operatorname{Hom}_{P \otimes \Gamma}\left(A, \operatorname{Hom}_{\Sigma}\left(B, C^{\prime \prime}\right)\right) \rightarrow 0 .
\end{aligned}
$$

Then, the isomorphism (2) gives an exact sequence

$$
0 \rightarrow \operatorname{Hom}_{\Gamma \otimes \Sigma}\left(A \otimes_{P} B, C^{\prime}\right) \rightarrow \operatorname{Hom}_{\Gamma \otimes \Sigma}\left(A \otimes_{P} B, C\right) \rightarrow \operatorname{Hom}_{\Gamma \otimes \Sigma}\left(A \otimes_{P} B, C^{\prime \prime}\right) \rightarrow 0,
$$
which proves by Prop. 8 that $A \bigotimes_{P} B$ is $(\Gamma \otimes \Sigma, \Lambda \otimes T)$-projective.

Similarly the second assertion is proved. 
COROLLARY 9.1. If $B_{P^{*} \otimes \Sigma}$ is $\left(P^{*} \otimes \Sigma, \Pi^{*} \otimes T\right)$-projective then $B$ is $(\Sigma, T)$-projective. If $C_{\Gamma \otimes P}$ is $(\Gamma \otimes P, \Lambda \otimes \Pi)$-injective then $C$ is $(\Gamma, \Lambda)$-injective.

ProOF. Replacing in Prop. $9 A, B, \Pi, P, \Lambda, \Gamma, T, P, \Sigma$ by $B, \Pi^{*}, P^{*}, T, \Sigma$, $K, K$, the first follows from Prop. 9. The second is similar.

COROLlARY 9.2. In the situation $\left({ }_{\Gamma} A, B_{\Sigma}\right)$ if $A$ is $(\Gamma, \Lambda)$-projective then $A \otimes_{K} B$ is $(\Gamma \otimes \Sigma, \Lambda \otimes T)$-projective. In the situation $\left({ }_{\Sigma} B, C_{\Gamma}\right)$ if $C$ is $(\Gamma, \Lambda)$ injective then $\operatorname{Hom}_{K}(B, C)$ is $(\Sigma \otimes \Gamma, T \otimes \Lambda)$-injective.

Proof is obtained by setting $P=\Pi=K$ in Prop. 9.

Corresponding to Prop. 3,

PROPOSITION 10. In the situation $\left(A_{P \otimes \Gamma},{ }_{P} B_{\Sigma}\right)$, let $X$ be a $(P \otimes \Gamma, \Pi \otimes \Lambda)$ projective resolution of $A$ (2. of $[4])$, and $Y$ a $\left(P^{*} \otimes \Sigma, \Pi^{*} \otimes T\right)$-projective resolution of $B$ as a right $P^{*} \otimes \Sigma$-module. If relative $\operatorname{Tor}_{n}^{(P, \mathrm{I})}(A, B)=0([4])$ for $n>0$ then $X \otimes_{P} Y$ is a $(\Gamma \otimes \Sigma, \Lambda \otimes T)$-projective resolution of $A \bigotimes_{P} B$.

PROOF. Since $Y$ is a $\left(\Sigma, T^{\top}\right)$-projective complex by Cor.9.1., $X \otimes_{r} Y$ is $(\Gamma \otimes \Sigma, \Lambda \otimes T)$-projective by Prop.9. From Cor.9.1. $X$ and $Y$ are $(P, \Pi)$ projective, thus $X$ and $Y$ are $(P, \Pi)$-projective resolutions of $A$ and $B$, hence

$$
H_{n}\left(X \otimes_{P} Y\right)=\operatorname{Tor}_{n}^{(P, I)}(A, B)=0 \text { for } n>0 \text {. }
$$

If we show that the sequence

$$
X_{1} \otimes_{P} Y_{0}+X_{0} \otimes_{P} Y_{1} \rightarrow X_{0} \otimes_{P} Y_{0} \rightarrow A \otimes_{P} B \rightarrow 0
$$

is exact, $X \otimes_{P} Y$ is a $(\Gamma \otimes \Sigma, \Lambda \otimes T)$-projective resolution of $A \bigotimes_{P} B$. This follows from the relative exactness of $\otimes_{P}$ just as [4] (the 15th line from the bottom of p. 251).

Now that we have obtained Prop. 10, we can introduce various products into our case.

To define generalised products $\wedge, \vee$, we may make only the following assumption corresponding to (i), (ii):

$$
\operatorname{Tor}_{n}^{\left(\Sigma, T^{T}\right)}\left(A, A^{\prime}\right)=0 \text { for } n>0, \quad{ }_{\triangle} A_{\Sigma},{ }_{\Sigma} A_{\Gamma}^{\prime},
$$

because Prop. 10 does not need the projectivess of operation rings.

That is in the situation $\left({ }_{\Lambda} A_{\Sigma},{ }_{\Lambda} C_{\Sigma},{ }_{\Sigma} A^{\prime}{ }_{\Gamma},{ }_{\Sigma} C^{\prime}{ }_{\Gamma}\right)$ with $\bar{\Lambda} \rightarrow \Lambda, \bar{\Gamma} \rightarrow \Gamma, \bar{\Sigma} \rightarrow \Sigma$, $\vee: \operatorname{Ext}_{\left(\Lambda \otimes \Sigma^{*}, \bar{\Lambda} \otimes \Sigma^{*}\right)}(A, C) \otimes \operatorname{Ext}_{\left(\bar{\Sigma} \otimes \Gamma^{*}, \bar{\Sigma} \otimes \Gamma^{*}\right)}\left(A^{\prime}, C^{\prime}\right) \rightarrow \operatorname{Ext}_{\left(\Lambda \otimes \Gamma^{* *}, \bar{\Lambda} \otimes \Gamma^{* *}\right)}\left(A \otimes_{\Sigma} A^{\prime}, C \otimes_{\Sigma} C^{\prime}\right)$, in the situation $\left({ }_{\Lambda} A_{\Sigma},{ }_{\Lambda} C_{\Sigma},{ }_{\Sigma} A^{\prime}{ }_{\Gamma},{ }_{\Gamma} C_{\Sigma}^{\prime}\right)$

$$
\begin{aligned}
& \wedge: \operatorname{Tor}^{\left(\Lambda \otimes \Gamma^{*}, \bar{\Lambda} \otimes \bar{\Gamma}^{*}\right)}\left(\operatorname{Hom}_{\Sigma}\left(C, C^{\prime}\right), A \otimes_{\Sigma} A^{\prime}\right) \rightarrow
\end{aligned}
$$

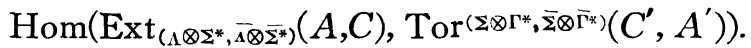

By setting $\Lambda=\Sigma=\Gamma=A$ in the above, since $\operatorname{Tor}_{n}^{(\Lambda, \Sigma)}(\Lambda)=$,0 for $n>0$ by Prop. 6 and 7, which shows as usual that the assumption(iii) is satisfied, we have, corresponding to (9), (13), 


$$
\begin{aligned}
& \vee: \operatorname{Ext}_{\left(\Lambda \otimes \Lambda^{*}, \bar{\Lambda} \otimes \Sigma^{*}\right)}(\Lambda, C) \otimes \operatorname{Ext}_{\left(\Lambda \otimes \Lambda^{*}, \bar{\Sigma} \otimes \bar{\Gamma}^{*}\right)}\left(A^{\prime}, C^{\prime}\right) \rightarrow \operatorname{Ext}_{\left(\Lambda \otimes \Lambda^{*}, \bar{\Lambda} \otimes \bar{\Gamma}^{*}\right)}\left(A^{\prime}, C \otimes_{\Lambda} C^{\prime}\right),
\end{aligned}
$$

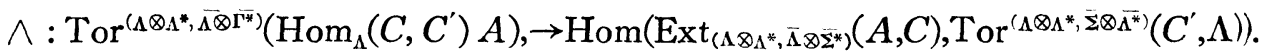

Thus in the same way as by (16), (17) for two-sided $\Lambda$-modules $A, C, F$ we have products

(40) U: $H_{\left(\Lambda \otimes \Lambda^{\prime \prime}, \bar{\Lambda} \otimes \bar{\Sigma}^{*}\right)}^{p}\left(\Lambda, \operatorname{Hom}_{\Lambda}(A, C)\right) \otimes \operatorname{Ext}_{\left(\Lambda \otimes \Lambda^{*}, \bar{\Sigma} \otimes \bar{\Gamma}^{*}\right)}^{q}(F, A) \rightarrow \operatorname{Ext}_{\left(\Lambda \otimes \Lambda^{*}, \Lambda \otimes \overline{1}^{*}\right)}^{p+q}(F, C)$,

(41) $\cap: \operatorname{Tor}_{p+q}^{\left(\Lambda \otimes \Lambda^{*}, \Lambda \otimes \bar{\Gamma}^{*}\right)}(C, F) \rightarrow \operatorname{Hom}\left(\operatorname{Ext}_{\left(\Lambda \otimes \Lambda^{*}, \bar{\Lambda} \otimes \bar{\Sigma}^{* *}\right)}^{p}(F, A), H_{q}^{\left(\Lambda \otimes \Lambda^{*}, \Sigma \bar{\Sigma} \otimes \Gamma^{*}\right)}\left(\Lambda, C \bigotimes_{\Lambda} A\right)\right)$, where relative functors are concerned to the homomorphisms of the second algebras in parentheses. Just as (18), (19), by using the identity $j$ of $A$,

By setting $\bar{\Sigma}=\bar{\Gamma}$ in (42) and $\bar{\Sigma}=\bar{\Lambda}$ in (43) we have

$$
\begin{aligned}
& \cup j: H_{\left(\Lambda \otimes \Lambda^{*}, \bar{\Lambda} \otimes \bar{\Sigma}^{*}\right)}^{p}\left(\Lambda, \operatorname{Hom}_{\Lambda}(A, C)\right) \rightarrow \operatorname{Ext}_{\left(\Lambda \otimes \Lambda^{*}, \Lambda \otimes \bar{\Gamma}^{*}\right)}^{n}(A, C), \\
& \left.\cap j: \operatorname{Tor}_{p}^{\left(\Lambda \otimes \Lambda^{*}, \overline{\bar{I}} \otimes \bar{\Gamma}^{*}\right)}(C, A) \rightarrow H_{p}^{\left(i \Delta \otimes \Lambda^{*}, \bar{\Sigma} \otimes \bar{\Gamma}^{*}\right)}\left(\Lambda, C \otimes_{\Lambda} A\right)\right)
\end{aligned}
$$

and in the case $p=0$ these are isomorphisms as Prop. 4.

Corresponding to Prop. 5, it holds without any restriction for $A$,

PROPOSITION 11. $\cup j$ and $\cap j$ are isomorphisms.

PROOF. Let $0 \rightarrow C \rightarrow Q \rightarrow N \rightarrow 0$ be an exact left $\left(\Lambda \otimes \bar{\Lambda}^{*}, \bar{\Lambda} \otimes \bar{\Gamma}^{*}\right)$ sequence with $Q$ leff $\Lambda \otimes \Lambda^{*}$-injective, then the sequence

$$
0 \rightarrow \operatorname{Hom}_{\Lambda}(A, C) \rightarrow \operatorname{Hom}_{\Lambda}(A, Q) \rightarrow \operatorname{Hom}_{\Lambda}(A, N) \rightarrow 0
$$

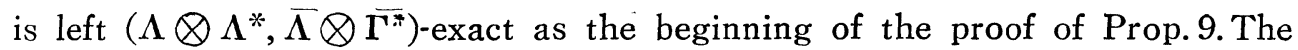
remainder is the same as Prop. 5.

Now that we have obtained Prop. 11, by changing " $\Lambda \otimes \Lambda^{*}$ " to " $\left(\Lambda \otimes \Lambda^{*}\right.$, $\left.\bar{\Lambda} \otimes \bar{\Gamma}^{*}\right)$ we shall get the similar results as below Prop. 5 in the section $1 \mathrm{thr}$ oughout. In Theorem 1, we do not need the restriction that $A$ is projective. In Cor. 1. 1. holds without that $\Lambda$ is projective, because $\Lambda$ is always $\left(\Lambda \otimes \Lambda^{*}\right.$, $\Lambda \otimes \Gamma^{*}$ )-projective by Prop. 6 . and 7 .

Now let $\Lambda, \bar{\Lambda}, \bar{\Gamma}$ be $K$-algebras with $K$-homomorphisms $\bar{\Lambda} \rightarrow \bar{\Lambda}$ and $\bar{\Gamma} \rightarrow$ $\Lambda$, and $C$ be a two-sided $\Lambda$-module. We shall define $(\bar{\Lambda}, \bar{\Gamma})$-extension over $\Lambda$ with kernel $C$ as an exact $\left(\Lambda \otimes \Lambda^{*}, \bar{\Lambda} \otimes \Gamma^{*}\right)$-sequence

$$
C \stackrel{g}{\longrightarrow} \Gamma \stackrel{f}{\longrightarrow} \Lambda
$$

where $\Gamma$ is a $K$-algebra, $f$ is a $K$-alge ra epimorphism, $g$ is a monomorphism of $K$-modules and

$$
g(\lambda c)=\gamma_{1}(g c), g(c \lambda)=(g c) \gamma_{2}, \quad c \in C, \gamma \in \Gamma, \lambda=f r \in \Lambda .
$$

As usual, we define the equivalence of extentions and obtain the analogy 
of Theorem 2, where $S(\Lambda)$ is the modified relative standard complex (p. 254 below of [4].), and an inessential extension is a direct sum of two $K$-algebras as $\bar{\Lambda} \bigotimes \overline{\boldsymbol{\Gamma}}^{*}$-rings. Then the same as Theorem 2 holds just as usual.

Since the relative standard complex may be normalised as easily seen in the same way as $[1]$, ([5]), we can define $X$ as an induced $\bar{\Lambda}-\bar{\Gamma}$-module as (28). In this manner Theorem 3 holds without restrictions for $\Lambda$, so Cor.3.1.

If $\Lambda=\bar{\Lambda}=R$ a $K$-algebra, $\bar{\Gamma}=S$ a $K$-subalgebra of $R$ in the above, we obtain the facts concerning the relative Hochschild groups. For these groups in the remainder we shall show the analogy to Theorem 4 without proof, which may be obtained easily by following the proof of Theorem 4 .

We shall represent $R, S$ as residue class rings of a $K$-free algebra $F$ with kernels $M, N$ which are ideals of $F$, and denote $C=M / M^{2}, \Gamma=F / M^{2}$. If $R$ has a basis over $S$, we have a $(R, S)$-extension over $R$ with kernel $C$

$$
0 \rightarrow C \rightarrow \Gamma \rightarrow R \rightarrow 0,
$$

With notation of [4], we have

THROREM 4'. Let $\xi$ be the relative cohomology class determined by $(\Sigma)$. The following isomorphisms hold for $p>0$,

$$
\begin{aligned}
& \left.H^{p}\left(R, S, \operatorname{Hom}_{R}(C, A)\right) \approx H^{p_{+2}}(R, S, A)\right) \\
& H_{p+2}(R, S, A) \approx H_{p}\left(R, S, A \otimes_{R} C\right)
\end{aligned}
$$

given by the modified products $h \cup \xi, h^{\prime} \cap \xi$.

For $p=0$, we have exact sequences

$$
\begin{aligned}
& \operatorname{Hom}_{R \otimes R^{*}}(\Gamma, A) \rightarrow \operatorname{Hom}_{R \otimes R^{*}}(C, A) \rightarrow H^{2}(R, S, A) \rightarrow 0, \\
& 0 \rightarrow H_{2}(R, S, A) \rightarrow A \bigotimes_{R} C \rightarrow A \bigotimes_{R} \Gamma .
\end{aligned}
$$

REMARK. $\operatorname{Hom}_{R}(C, A)$ rnay be considered as the module $\operatorname{Hom}_{F}(M, A)$ of all right $F$-homomorphisms of $M$ into $A$ regarded as $F$-modules induced by the $\operatorname{map}: F \rightarrow R$.

At the end I wish to express my hearty thanks to Professor T.Tannaka for his kind guidance during my researches.

\section{REFERENCES}

[1] H. CArtan and S. Eilenberg, Homological algebra, Princeton, 1956.

[2] S. Eilenberg, and S. MAClane, Cohomology theory in abstract groups I, Ann. of Math. 48(1947), 51-78.

[3] G. HocHSCHILD, On the cohomoloy groups of an associative algebras. Ann. of Math. $46(1945)$, 58-67.

[4] G. HochSCHILD, Relative homological algebra, Trans. A. M. S. 82(1956) 246-269.

[5] H. YAMASAKI, The reduction theorem of the relative cohomology group in algebra, and its application. Annual Raport of the Iwate Univ. 14(1958)1-13 (in Japanese).

Iwate University. 